\title{
Crecimiento radial de la especie endémica Nothomyrcia fernandeziana (Hook. \& Arn.) Kausel y la invasora Aristotelia chilensis (Molina) Stuntz en la isla Robinson Crusoe, Archipiélago de Juan Fernández, Chile
}

\section{Radial growth of the endemic species Nothomyrcia fernandeziana (Hook. \& Arn.) Kausel and the invasive species Aristotelia chilensis (Molina) Stuntz in Robinson Crusoe Island, Juan Fernandez Archipelago, Chile}

\author{
Moisés Rojas-Badilla*1 ${ }^{*}$, Carlos Le Quesne ${ }^{1}$, Claudio Álvarez ${ }^{1}$, Gonzalo Velásquez-Álvarez ${ }^{1}$ \& \\ VICENTE ROZAS ${ }^{1,2}$ \\ 'Laboratorio de Dendrocronología y Cambio Global, Instituto de Conservación Biodiversidad y Territorio, Facultad de Ciencias \\ Forestales y Recursos Naturales, Universidad Austral de Chile, Casilla 567, Valdivia, Chile. \\ ${ }^{2}$ Área de Botánica, Campus Duques de Soria, Universidad de Valladolid, 42004 Soria, España. \\ *moisese.rojasbadilla@gmail.com
}

\begin{abstract}
RESUMEN
Nothomyrcia fernandeziana (Hook. \& Arn.) Kausel es una especie endémica de los bosques de la isla Robinson Crusoe (RC), Archipiélago de Juan Fernández, Chile. Aristotelia chilensis (Molina) Stuntz es una especie invasora introducida en la isla a mediados del siglo XIX que compite con las especies endémicas, poniendo en riesgo su conservación. Los patrones de crecimiento radial permiten inferir estrategias biológicas de las plantas leñosas como longevidad, tolerancia a la sombra y capacidad competitiva, características de gran importancia para los programas de conservación. Los objetivos de este trabajo fueron caracterizar los anillos de crecimiento de ambas especies y estudiar sus patrones de crecimiento con el objeto de aportar conocimiento base que contribuya a la conservación de los bosques endémicos de RC. Se realizó el estudio anatómico y dendrocronológico de los anillos de crecimiento en secciones transversales del tronco de ambas especies en $\mathrm{RC}$, así como de $A$. chilensis en su área de distribución natural en Chile continental. La madera de ambas especies presentó anillos de crecimiento bien diferenciados. N. fernandeziana mostró un crecimiento juvenil reducido, árboles longevos y alta capacidad de liberación del crecimiento (aumento superior al 100\% del promedio de los valores de ancho de anillos de los 10 años previos al evento). A. chilensis, sin embargo, presentó una alta tasa de crecimiento durante su ontogenia y escasa longevidad, comportándose como especie pionera en $\mathrm{RC}$ al igual que en su área de distribución natural. Sobre la base de estos resultados, se recomienda actuar favoreciendo el establecimiento de $N$. fernandeziana a la sombra de A. chilensis, propiciando la gradual sustitución de la especie invasora por especies endémicas de RC.
\end{abstract}

Palabras clave: Anillos de crecimiento, restauración, dendroecología, conservación.

\begin{abstract}
Nothomyrcia fernandeziana (Hook. \& Arn.) Kausel is an endemic tree species from Robinson Crusoe Island (RC), Juan Fernández Archipelago, Chile. Aristotelia chilensis (Molina) Stuntz, is an invasive species introduced in the island since the mid XIX century that compete with endemic species and threatens their conservation. Radial growth patterns can reveal biological strategies of woody plants, such as longevity, shade tolerance and competitive capacity, which are of special relevance for conservation programmes. The objectives of this work were to characterize the tree rings of both species and to study their radial growth patterns, in order to obtain basic knowledge for the conservation of endemic forests in RC. We studied wood anatomy and dendrochronology for stem cross sections of both species in $\mathrm{RC}$, and also of $A$. chilensis within its natural distribution range in continental Chile. The wood of both species exhibited well-differentiated tree rings. $N$. fernandeziana showed reduced juvenile growth rates, long-lived trees and high capacity to release its growth (increase greater than $100 \%$ of the average ring-width for the 10 years before the event). A. chilensis, by contrast, showed large growth rates during their complete lifespan but reduced longevity, a typical behaviour of a pioneer species, as well as in their natural range. Based on these results, it is suggested to promote the establishment of $N$. fernandeziana under the canopy of $A$. chilensis, favouring the gradual replacement of the invasive species by the endemic species in Robinson Crusoe.
\end{abstract}

KEYWORDS: Tree rings, restoration, dendroecology, conservation. 


\section{INTRODUCCIÓN}

El archipiélago de Juan Fernández alberga ca. 62\% de especies vegetales endémicas (Vargas \& Faúndez 2010). Robinson Crusoe (RC), también llamada Más a Tierra, es una de las islas principales del archipiélago y se ubica a unos $650 \mathrm{~km}$ al oeste de la línea costera de Chile continental, a la latitud de San Antonio (33³6’). Su vegetación incluye una flora única particularmente amenazada por las actividades humanas y la competencia con especies de plantas invasoras (Vargas et al. 2010b). Entre las especies arbóreas endémicas, la más común es Nothomyrcia fernandeziana (Hook. \& Arn.) Kausel, la luma de Juan Fernández, árbol siempreverde que alcanza 20 a $25 \mathrm{~m}$ de altura. Esta especie pertenece a la familia Myrtaceae y representa un género monotípico endémico de RC (Murillo-Aldana \& Ruiz 2011), que se encuentra clasificado como vulnerable (World Conservation Monitoring Centre 1998). La madera de $N$. fernandeziana ha sido descrita como un leño muy duro, de apariencia homogénea y color claro, sin distinción entre albura y duramen, y con anillos de crecimiento que pueden alcanzar los 0,3 cm de ancho (Johow 1896). N. fernandeziana domina la mayor parte de la cobertura boscosa de RC (Johow 1896, Ricci et al. 2008, Vargas et al. 2014). No obstante, la presencia de especies introducidas e invasoras como Aristotelia chilensis (Molina) Stuntz, registrada en la isla desde mediados del siglo XIX, amenaza la conservación de estos bosques (Johow 1896, Dirnböck 2003). Basándose en el carácter de especie pionera debido a su eficiente diseminación de semillas, y a su capacidad de rebrote de tocón (Muñoz \& González 2006, Bernardello et al. 2011), se ha planteado que la competencia directa de A. chilensis por recursos puede provocar la muerte de individuos de $N$. fernandeziana por sequía y sofocamiento (Johow 1896). Ello, en función de la alta densidad de las poblaciones que han logrado establecerse en los bosques originales de RC (Danton 2004). Sin embargo, hasta ahora no se dispone de datos relativos a sus respectivas tasas de crecimiento, indicadoras del vigor y desarrollo potencial de ambas especies en la isla. De ahí la relevancia de investigar los patrones de crecimiento de estas especies y las edades máximas que pueden alcanzar. Dichas características básicas pueden ser claves para entender los patrones temporales de establecimiento y crecimiento de las especies arbóreas presentes en RC y así proyectar posibles escenarios futuros en los bosques de la isla.

Utilizando técnicas dendrocronológicas para la identificación y descripción de los anillos de crecimiento, a través de cortes transversales del leño, en este trabajo se pretende evaluar el potencial de Nothomyrcia fernandeziana (endémica) y Aristotelia chilensis (introducida) para realizar investigaciones relacionadas al ancho de anillos en RC. Esto contribuiría al estudio detallado de la dinámica de los bosques de la isla, el desplazamiento y/o reemplazo potencial de las especies, y podría aportar conocimiento base para la futura conservación de los bosques endémicos de RC. Como objetivos específicos se plantean: 1) identificar y describir los anillos de crecimiento de ambas especies a nivel anatómico y evaluar su potencial para construir series de ancho de anillos confiables, y 2) cuantificar y comparar el incremento medio anual en radio de ambas especies y analizar sus patrones de crecimiento.

\section{MATERIAL Y MÉTODOS}

ÁreA DE ESTUdio

El Archipiélago de Juan Fernández se ubica a $33^{\circ} \mathrm{S}$ en el Océano Pacífico y está compuesto de tres islas, Alejandro Selkirk, Santa Clara y Robinson Crusoe. Las islas son de origen volcánico y en el caso de RC originada hace ca. 4 millones de años (Stuessy et al. 1997). RC presenta un clima mediterráneo con marcada influencia oceánica y precipitaciones totales anuales que rondan en promedio los $950 \mathrm{~mm}$. Alrededor del $45 \%$ de las precipitaciones se concentran en invierno y disminuyen al $10 \%$ en los meses de verano. Las temperaturas mínimas medias y máximas medias mensuales alcanzan a 10,1 y $21,8{ }^{\circ} \mathrm{C}$, respectivamente, con una media anual de $15,2{ }^{\circ} \mathrm{C}$ (Hajek \& Espinoza 1987).

La Myrtiselva (bosque dominado por Myrtaceae) en Plazoleta El Yunque, sector del que proviene el material leñoso analizado, se encuentra compuesta por las especies arbóreas endémicas Nothomyrcia fernandeziana, Fagara mayu Engl. y Drimys confertifolia Phil. (Greimler et al. 2002, Vargas et al. 2010a, 2010b), donde $N$. fernandeziana es la especie más frecuente (800 árboles ha-1) y con mayor mortalidad (45 árboles ha-1) (Vargas et al. 2010a). La dinámica de claros producto de la caída de árboles dominantes es el principal factor que promueve la regeneración de las especies arbóreas, pero da lugar a la invasión de especies exóticas intolerantes a la sombra como A. chilensis, que compiten con las especies endémicas por su nicho de regeneración (Smith-Ramírez et al. 2013).

\section{RECOLECCIÓN Y PROCESAMIENTO DE MUESTRAS}

El muestreo, de carácter exploratorio, contempló 6 secciones transversales completas (rodelas con centro y corteza) extraídas de individuos muertos de $N$. fernandeziana y $A$. chilensis resultantes del desarraigo natural de árboles por los vientos oceánicos. A partir de cortes transversales, se describieron las características anatómicas de la madera y de los anillos de crecimiento de ambas especies, basados en elementos anatómicos básicos como disposición $\mathrm{y}$ tamaño de los vasos, tipos de radios leñosos y presencia de parénquima. Estos elementos anatómicos y su disposición en los anillos anuales de crecimiento se analizaron para facilitar la identificación de los límites entre una temporada de crecimiento y la siguiente. 
Con el objetivo de conocer las tasas de crecimiento anual de una especie poco estudiada como A. chilensis, y así obtener valores de referencia que permitan comparar con los valores alcanzados en los bosques de $\mathrm{RC}$, se recolectaron muestras de 6 individuos dentro del rango de distribución natural de A. chilensis. El lugar de la recolecta fue el Arboretum perteneciente a la Universidad Austral de Chile ubicado en la Isla Teja, Valdivia, ca. $40^{\circ} \mathrm{S}$ (Hechenleitner \& Zamorano 2005). Los individuos fueron recolectados en fragmentos de bosque nativo secundario dominado por Nothofagus obliqua (Mirb.) Oerst., Laurelia sempervirens (Ruiz \& Pav.) Tul. y Aextoxicon punctatum Ruiz et Pav., entre otras arbóreas. Este bosque nativo tiene objetivos de conservación y recuperación del bosque original de la Isla Teja, no perteneciendo los árboles muestreados a las colecciones del Arboretum, y donde A. chilensis regenera y crece de forma natural.

Las rodelas se pulieron con lijas de granulometría decreciente (i.e. de la más gruesa a la más fina, entre 80 y 800 granos $\mathrm{cm}^{2}$ ), para luego identificar los límites de los anillos de crecimiento y determinar la edad de los ejemplares bajo microscopio estereoscópico $(<63 \times)$. La delimitación de los anillos de crecimiento de ambas especies se basó en las principales características anatómicas en corte transversal del leño. Es importante destacar que debido al limitado número de ejemplares analizados y al desconocimiento de las fechas de muerte de cada individuo no fue posible establecer el año exacto en que se formó cada anillo (Fritts \& Swetnam 1989). Sin embargo, fue posible registrar y medir las secuencias completas de ancho de anillos de crecimiento anual y calcular las edades y las tasas de incremento radial. La medición del ancho de anillos de cada muestra se realizó con un carro de medición Velmex ${ }^{\circledR}$ con precisión de 0,001 $\mathrm{mm}$.

\section{CRECIMIENTO RADIAL Y EXCENTRICIDAD}

Todas las rodelas obtenidas poseían médula y corteza, lo que permitió cuantificar el crecimiento inicial a lo largo de su período de vida y calcular el número de años que tenía cada individuo al momento de morir. La edad individual se cuantificó como el número total de anillos existentes entre la médula y la corteza en cada rodela, obteniéndose la edad real de cada individuo a la altura de muestreo.

Tomando como referencia la distancia mayor médulacorteza de cada muestra, se trazaron y midieron cuatro radios ortogonales entre sí, con los que se calculó el radio promedio para luego dividirlo por el total de años de la muestra, obteniendo el crecimiento medio anual o incremento radial medio. La tasa de crecimiento anual máxima se calculó dividiendo el radio mayor por el total de años de la muestra. La tasa de crecimiento inicial se obtuvo promediando el ancho de los 10 anillos centrales de cada rodela.

La forma de las rodelas de ambas especies se determinó mediante un índice de excentricidad del crecimiento. Este procedimiento busca cuantificar en qué medida la forma de cada rodela se desvía de un crecimiento idealmente concéntrico, en que la médula se encontraría en el centro geométrico de cada rodela. El índice de excentricidad del crecimiento (E) fue definido como $\mathrm{E}=[1-(\mathrm{m} / \mathrm{M})] \cdot 100$, donde $\mathrm{m}$ y $\mathrm{M}$ son, respectivamente, las longitudes del radio menor y mayor entre la corteza y la médula a lo largo de la circunferencia completa de la rodela (Rozas 2003). El índice E varía entre 0 si el crecimiento fue concéntrico $(\mathrm{m}=\mathrm{M})$, y 100 si el crecimiento fue totalmente excéntrico $(\mathrm{m}=0)$.

La existencia de diferencias significativas en la edad, el área y el perímetro de las rodelas, la excentricidad del crecimiento, y las tasas de crecimiento media, máxima e inicial entre $N$. fernandeziana, A. chilensis en RC y $A$. chilensis en Valdivia, fue comprobada mediante la prueba no paramétrica de Kruskal-Wallis. Se utilizó la prueba post hoc de comparaciones múltiples de Dunnett para diferenciar aquellos grupos que difirieron significativamente entre sí. Los análisis estadísticos se realizaron con el software SPSS v.15.0.

La presencia de liberaciones abruptas del crecimiento en las series de ancho de anillos, las cuales se deben a la aparición de claros en el dosel arbóreo, se detectó mediante el filtro de porcentaje del cambio en el crecimiento (PCC) (Nowacki \& Abrams 1997). Las liberaciones del crecimiento se detectaron mediante la fórmula $\mathrm{PCC}=[(\mathrm{M} 2$ - M1) / M1] · 100, siendo M1 y M2 las tasas medias de crecimiento de los 10 años previos y subsiguientes a cada anillo considerado, respectivamente. Este procedimiento establece la relación entre la tasa media de crecimiento de una década y la media de la década anterior. Una liberación abrupta fue definida como un aumento en al menos el $100 \%$ del crecimiento cuando fueron comparadas las tasas medias en períodos consecutivos de 10 años.

\section{RESULTADOS}

DESCRIPCIÓN DE LOS ANILLOS DE CRECIMIENTO

Las rodelas examinadas de $N$. fernandeziana presentaron tonalidades de color café claro y oscuro producto de la degradación parcial de la madera por hongos, que además del crecimiento marcadamente lobulado dificultaron en cierta medida la visualización de los límites entre anillos (Fig. 1A). Sin embargo, se logró diferenciar inequívocamente los anillos de crecimiento. Nothomyrcia fernandeziana presentó anillos de crecimiento con vasos solitarios, radios leñosos uni- y multiseriados, con abundante parénquima (células más claras), y un límite definido entre la madera tardía más oscura y con escasa presencia de vasos, y la madera temprana más clara y con abundantes vasos distribuidos homogéneamente (Fig. 1B). Los límites entre anillos de crecimiento consecutivos se distinguieron por la tonalidad 
más oscura de la madera tardía y su contraste con la madera temprana del siguiente anillo.

El color café claro de la madera de A. chilensis y el contraste de la madera temprana clara con la tardía oscura, además de la forma relativamente concéntrica del crecimiento (Fig. 2A), permitieron reconocer con facilidad bajo microscopio los límites entre anillos consecutivos. Aristotelia chilensis presentó radios leñosos uniseriados y vasos solitarios y múltiples dispuestos en filas de hasta 5 vasos alineados en dirección radial, con paredes de contacto aplanadas (Fig. 2B). En cada temporada de crecimiento, los vasos disminuyen en tamaño desde el inicio de la madera temprana hacia el final del periodo vegetativo. Existen límites marcados entre la madera temprana y la madera tardía, siendo más ancha y clara la primera y más estrecha y oscura esta última (Fig. 2B).
PATRONES DE CRECIMIENTO RADIAL

Las tasas y los patrones de crecimiento de las especies estudiadas se calcularon a partir de 3.264 anillos de crecimiento de $N$. fernandeziana, 340 de $A$. chilensis de RC y 468 de $A$. chilensis de Valdivia. Las rodelas estudiadas difirieron entre especies en su edad y excentricidad del crecimiento (Tabla 1). Fueron más longevos y con mayor perímetro los árboles de $N$. fernandeziana que los de $A$. chilensis en todos los casos. Los árboles de $N$. fernandeziana mostraron mayor excentricidad en el crecimiento que $A$. chilensis en Valdivia, pero no fueron más excéntricos que los árboles de RC de esta misma especie (Tabla 1).

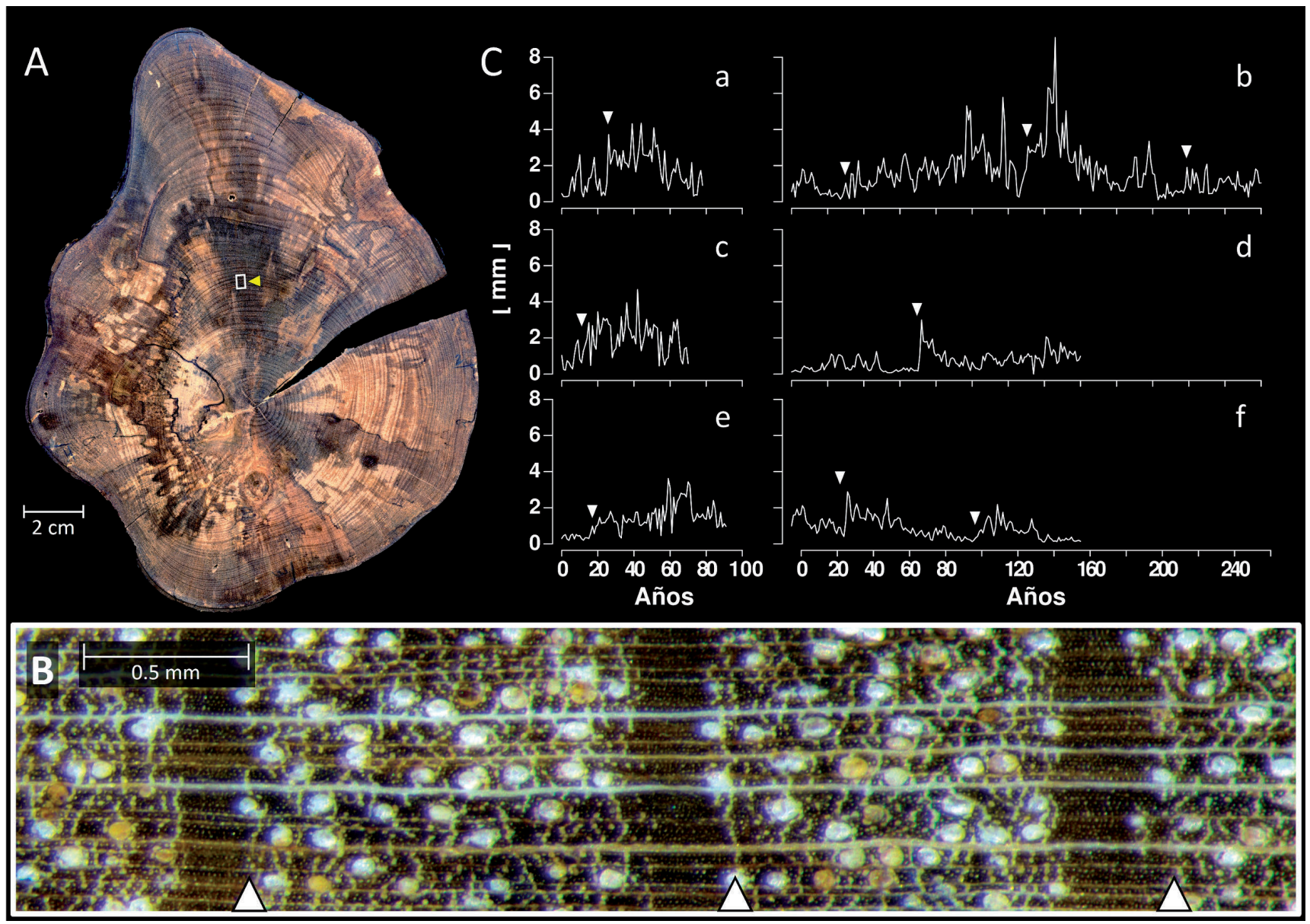

Figura 1. A) Sección transversal de tronco de N. fernandeziana de RC, con madera decolorada a consecuencia de la descomposición parcial, mostrando su crecimiento lobulado característico. El rectángulo y la flecha indican el área de visualización de los anillos de crecimiento en la Figura B. B) Anillos de crecimiento de $N$. fernandeziana con clara diferenciación anatómica entre leño temprano, con abundantes vasos, y leño tardío apenas sin vasos. C) Gráficos de ancho de anillos de las muestras de N. fernandeziana recolectadas en RC, donde el eje "y" representa el crecimiento en milímetros y el eje " $\mathrm{x}$ " la edad de las muestras, desde médula a corteza. / A) Cross section of $N$. fernandeziana stem from RC, with partially decolorated wood and a typical lobulated growth. Rectangle and arrow indicate the area shown in panel B. B) Tree rings of $N$. fernandeziana with anatomical differentiation between the earlywood with abundant vessels, and the latewood without vessels. C) Tree ring width series of $N$. fernandeziana from RC; " $y$ " axis is growth rate in $\mathrm{mm}$, " $\mathrm{x}$ " axis is tree age, from pith to bark. 


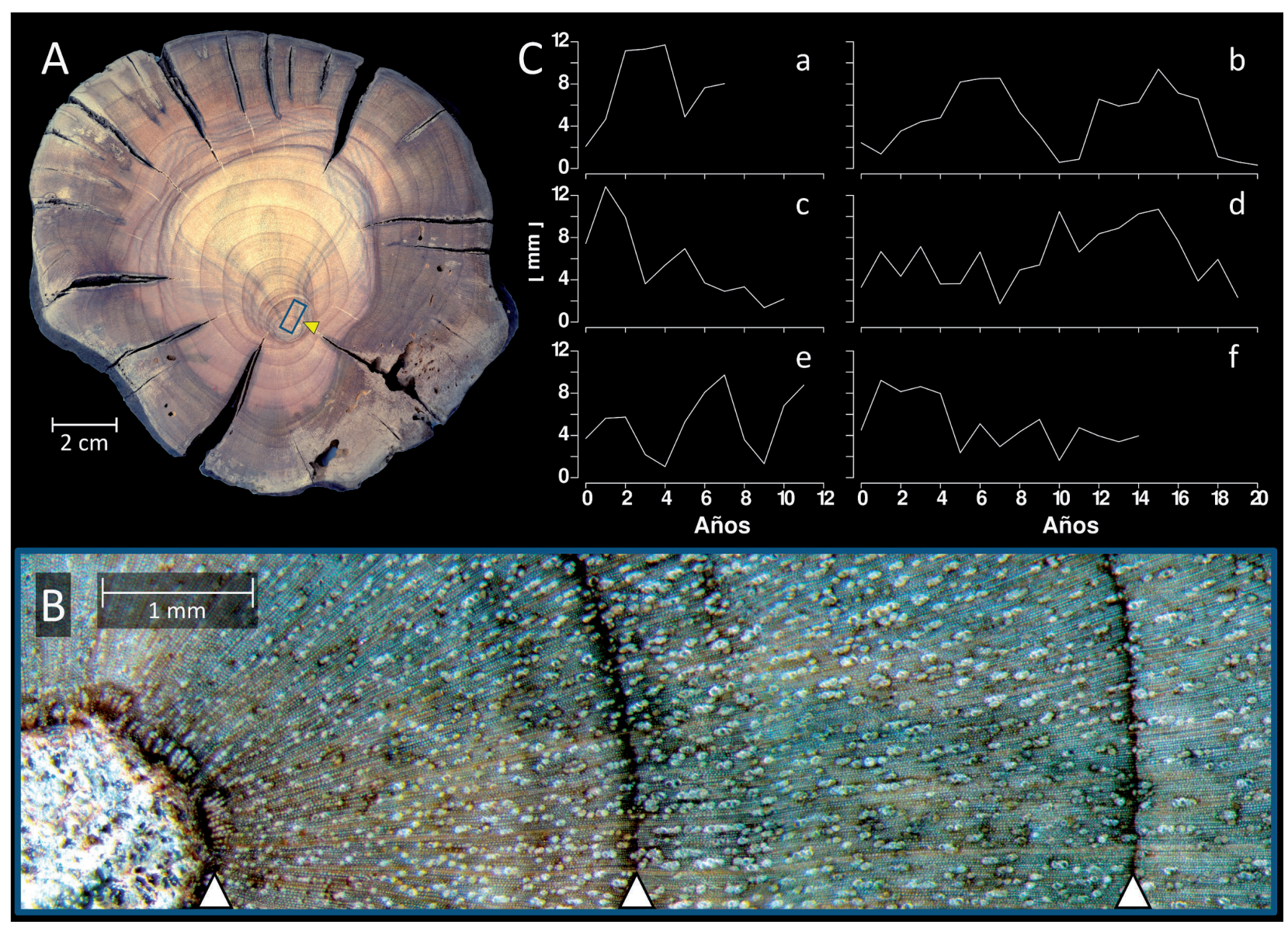

Figura 2. A) Sección transversal de tronco de A. chilensis de RC, mostrando crecimiento relativamente concéntrico, aunque levemente excéntrico durante el período de crecimiento juvenil. El rectángulo y la flecha indican el área de visualización de los anillos de crecimiento de la figura B. B) Anillos de crecimiento de A. chilensis bien delimitados por el contraste de color entre la madera temprana ancha y clara, y la madera tardía más estrecha y oscura. Se muestran los dos anillos centrales más próximos a la médula del tallo. C) Gráficos de ancho de anillos de las muestras de $A$. chilensis recolectadas en $\mathrm{RC}$, donde el eje "y" representa el crecimiento en milímetros y el eje "x" la edad de las muestras, desde médula a corteza. / A) Cross section of $A$. chilensis stem from RC, with relatively concentric growth, although moderately eccentric during the juvenile stage. Rectangle and arrow indicate the area shown in panel B. B) Tree rings of $A$. chilensis with anatomical differentiation between the wide and pale earlywood, and the narrow and dark latewood. The two central rings, adjacent to the pith, are shown. C) Tree ring width series of A. chilensis from RC; " $y$ " axis is growth rate in mm, " $\mathrm{x}$ " axis is tree age, from pith to bark.

Las curvas de crecimiento de todas las rodelas analizadas de $N$. fernandeziana presentaron liberaciones bruscas en sus anillos de crecimiento, con ancho de anillos que varían entre 0,07 y $9 \mathrm{~mm}$. La serie más longeva de $N$. fernandeziana, con 250 años de edad, mostró tres liberaciones bruscas del crecimiento mayores que $100 \%$ de PCC (Fig. 1C-b). La primera comenzó a los 27 años del árbol, la siguiente a los 128 años, y la última a los 212 años, indicando que los árboles maduros de esta especie conservaron gran capacidad de reacción a la apertura de claros en el dosel. Sin embargo, los crecimientos juveniles iniciales fueron muy inferiores a los registrados a partir de los 20 a 60 años de edad de los árboles, cuando aumentaron significativamente en mayor o menor medida dependiendo de la muestra. El periodo más prolongado de crecimiento reducido juvenil lo exhibió la segunda serie más longeva de nuestros registros, con un total de 161 años (Fig. 1C-d), que a la edad de 61 años experimentó la mayor liberación del crecimiento registrada, alcanzando un aumento del 594\% respecto a la tasa previa de crecimiento. Este resultado indicaría una notable capacidad de la especie para sobrevivir a la sombra y alcanzar el dosel del bosque. Los patrones de crecimiento de A. chilensis en $\mathrm{RC}$, sin embargo, no mostraron ninguna liberación brusca del crecimiento, sino una tasa de crecimiento elevada durante casi toda su vida, con edades máximas próximas a los 20 años (Fig. 2C).

Los resultados referentes a tasas de crecimiento indicaron diferencias significativas entre especies, siendo el crecimiento de $N$. fernandeziana claramente inferior al de $A$. chilensis, tanto en RC como en Valdivia (Tabla 1). La especie A. chilensis en RC creció anualmente en promedio un $706 \%$ más que $N$. fernandeziana y un $137 \%$ más que $A$. chilensis 
de Valdivia, aunque las tasas medias de crecimiento de $A$. chilensis en RCy en Valdivia no difirieron significativamente (Tabla 1). En cuanto al potencial de crecimiento máximo y al crecimiento inicial durante los 10 primeros años de vida, A. chilensis superó con creces a $N$. fernandeziana en ambos casos (Tabla 1). Las tasas de crecimiento máxima e inicial de $A$. chilensis en RC fueron un 465 y un $664 \%$ superiores, respectivamente, a las correspondientes tasas de crecimiento de $N$. fernandeziana. Aunque las tasas de crecimiento media, máxima e inicial de $A$. chilensis creciendo en $\mathrm{RC}$ fueron superiores a las registradas en esta especie creciendo en Valdivia, no existieron diferencias significativas entre las tasas de crecimiento de A. chilensis creciendo en $\mathrm{RC}$ y en Valdivia (Tabla 1).

TABLA 1. Diferencias entre edad, excentricidad del crecimiento y tasas de crecimiento (TC) media, máxima e inicial de $N$. fernandeziana y A. chilensis recolectadas en RC y en Valdivia. Los resultados muestran valores promedios $\pm \mathrm{SE}$ de 6 réplicas de individuos diferentes. Letras distintas representan diferencias significativas entre la variable considerada y la especie $(\mathrm{P}<0,05)$, utilizando la prueba de Dunnett. Para cada variable se muestran los estadísticos $\left(\chi^{2}, \mathrm{P}\right)$ de la prueba de Kruskal-Wallis para la comparación entre medias. / Differences in age, growth eccentricity, and mean, maximum and initial growth rates (TC) of $N$. fernandeziana and A. chilensis from RC and Valdivia. Results show mean values \pm SE of 6 replicates from different individuals. Different letters represent significant differences between variables and species $(\mathrm{P}<0.05)$ using Dunnett test. The statistics of Kruskal-Wallis test $\left(\chi^{2}, \mathrm{P}\right)$ are shown for every variable.

\begin{tabular}{|c|c|c|c|c|c|}
\hline VARIABLE & ESPECIE & UBICACIÓN & Media \pm SE & $\chi^{2}$ & $\mathrm{P}$ \\
\hline \multirow[t]{3}{*}{ Edad (años) } & N. fernandeziana & Robinson Crusoe & $136 \pm 28,3(a)$ & 11,73 & 0,002 \\
\hline & A. chilensis & Robinson Crusoe & $14 \pm 2,3(b)$ & & \\
\hline & A. chilensis & Valdivia & $19 \pm 3,9(b)$ & & \\
\hline \multirow[t]{3}{*}{ Excentricidad (\%) } & N. fernandeziana & Robinson Crusoe & $69,4 \pm 7,3(a)$ & 7,24 & 0,027 \\
\hline & A. chilensis & Robinson Crusoe & $48,9 \pm 5,3(\mathrm{ab})$ & & \\
\hline & A. chilensis & Valdivia & $42,4 \pm 4,8(b)$ & & \\
\hline \multirow[t]{3}{*}{ TC media (mm año-1) } & N. fernandeziana & Robinson Crusoe & $0,72 \pm 0,06(\mathrm{a})$ & 12,12 & 0,002 \\
\hline & A. chilensis & Robinson Crusoe & $5,15 \pm 0,76(b)$ & & \\
\hline & A. chilensis & Valdivia & $3,75 \pm 0,63(b)$ & & \\
\hline \multirow[t]{3}{*}{ TC máxima (mm año $\left.{ }^{-1}\right)$} & N. fernandeziana & Robinson Crusoe & $1,41 \pm 0,22(a)$ & 11,94 & 0.003 \\
\hline & A. chilensis & Robinson Crusoe & $6,58 \pm 0,78(b)$ & & \\
\hline & A. chilensis & Valdivia & $4,81 \pm 0,86(b)$ & & \\
\hline \multirow[t]{3}{*}{ TC inicial $\left(\mathrm{mm}\right.$ año $\left.{ }^{-1}\right)$} & N. fernandeziana & Robinson Crusoe & $0,84 \pm 0,19$ (a) & 12,31 & 0.002 \\
\hline & A. chilensis & Robinson Crusoe & $5,55 \pm 0,45(b)$ & & \\
\hline & A. chilensis & Valdivia & $4,31 \pm 0,51(b)$ & & \\
\hline
\end{tabular}

\section{DISCUSIÓN}

La anatomía de la madera se utiliza ampliamente en botánica para la clasificación filogenética de las diferentes especies (Díaz-Vaz 1979, Dias-Leme et al. 1995). En el caso de $N$. fernandeziana, los géneros más cercanos son Blepharocalyx, Ugni, Myrcianthes, Myrtus y Luma (Murillo-Aldana \& Ruiz 2011, Murillo-Aldana et al. 2012). Cabe destacar que la estructura anatómica de la madera es todavía desconocida para la gran mayoría de especies de la familia Myrtaceae (Denardi \& Cardoso 2005). Sin embargo, existen trabajos de especies tropicales pertenecientes a los géneros Blepharocalyx y Myrcianthes (Denardi \& Cardoso 2005, Rodrigues \& Cardoso 2010). Blepharocalyx salicifolius (Kunth) O. Berg y Myrcianthes cisplatensis (Camb.) O. Berg., ambas especies nativas de selvas tropicales, presentan una estructura anatómica de la madera muy similar a Nothomyrcia. Entre las características en común se encuentran principalmente la gran cantidad de vasos solitarios con distribución difusa y radios medulares 
muy abundantes y predominantemente uniseriados $\mathrm{y}$ biseriados. A pesar de las similitudes en cuanto a la anatomía y la cercanía filogenética, sólo $N$. fernandeziana presenta una madera tardía bien definida por la ausencia de vasos y parénquima, la que facilita notablemente el reconocimiento de los límites de los anillos en comparación con especies tropicales relacionadas.

En cuanto a la familia Elaeocarpaceae, las especies pertenecientes al género Aristotelia presentan anillos generalmente bien definidos. El elemento anatómico común más importante para el reconocimiento de los anillos de crecimiento en la madera de A. chilensis, $A$. serrata Oliv. y $A$. fruticosa Hook.f. es el color blanquecino a café claro de la madera temprana y café oscuro de la madera tardía (Rajni 1989). Esta característica, sumada a la baja densidad de vasos pequeños, solitarios y múltiples formando cadenas en dirección radial, permite identificar claramente los límites de cada anillo anual de crecimiento. No ocurre así con el género Elaeocarpus perteneciente a la misma familia, donde los anillos anuales no presentan un límite bien definido (Rajni 1989).

La madera de ambas especies estudiadas muestra excelentes propiedades anatómicas para el estudio de los anillos de crecimiento. Una clara diferenciación de los anillos anuales de la madera permite realizar estimaciones precisas de la edad de los árboles (Rozas 2003), y analizar en detalle los patrones de crecimiento individual. Esto contribuye a la obtención de conocimiento base sobre las características ecológicas de las especies, la dinámica forestal (regeneración, perturbaciones) y el estudio del impacto de las condiciones ambientales anómalas sobre el crecimiento de los árboles (Fritts \& Swetnam 1989, Brienen \& Zuidema 2006). Este tipo de análisis resulta muy útil para el diseño de estrategias de conservación de las especies leñosas (Swetnam et al. 1999, Allen et al. 2002).

El crecimiento marcadamente lobulado de $N$. fernandeziana genera el acuñamiento de los anillos y provoca la compresión, o en algunos casos ausencia, del crecimiento anual en ciertos sectores de las rodelas. Esta característica también se presenta en otras especies nativas de Chile (Rojas-Badilla et al. en revisión), resultando particularmente acentuado en las especies de la familia Podocarpaceae (Rozas et al. 2016). Consecuentemente, para realizar estudios sobre el crecimiento de esta especie no sería recomendable extraer sólo muestras de árboles vivos mediante taladros de incremento, debido al riesgo de tomar excesivas muestras con micro-anillos y/o anillos ausentes que dificulten e incluso impidan la identificación del número real de anillos de crecimiento (Speer 2010). Por otra parte, la alta densidad y dureza de la madera de $N$. fernandeziana dificulta en gran medida la extracción de testigos con taladro de incremento. Por ello, para futuros muestreos dendrocronológicos de $N$. fernandeziana en $\mathrm{RC}$ es recomendable recolectar secciones completas de un número suficientemente elevado de árboles muertos, junto con un número aceptable de testigos de árboles vivos. Todo ello teniendo en cuenta la elevada frecuencia de acuñamiento en los anillos y considerando que se trata de una especie endémica que sólo existe de forma natural en $\mathrm{RC}$, y por lo tanto su conservación es prioritaria. La especie $A$. chilensis, en cambio, presenta un crecimiento relativamente concéntrico y con lóbulos en su crecimiento poco acentuados. Considerando que se trata de una especie introducida en la isla y de carácter invasor, sería posible estudiar su dinámica poblacional y de crecimiento a partir de secciones transversales del leño tanto de árboles vivos como muertos.

En cuanto a las tasas de crecimiento anual, $N$. fernandeziana es sobrepasada ampliamente por $A$. chilensis, ya sean los árboles del continente o de RC. Vale decir que para alcanzar el diámetro de un A. chilensis de 20 años, a $N$. fernandeziana le tomarían ca. 140 años. Esto quiere decir que el potencial de crecimiento de la especie invasora supera ampliamente al potencial de crecimiento de la especie endémica, y que la especie invasora se comporta igual, en términos de dinámica del crecimiento, que en su área de origen.

A. chilensis se encuentra en la isla RC desde antes de 1864 (Johow 1896, Dirnböck et al. 2003), por lo que esta especie en la actualidad lleva al menos 152 años en el lugar. Las especies exóticas leñosas con mayor carácter invasor en RC son A. chilensis y Rubus ulmifolius Schott, las cuales se establecen densamente en claros del dosel arbóreo y áreas alteradas (Vargas et al. 2013, Arellano-Cataldo \& Smith-Ramírez 2016). Esta evidencia es coherente con los patrones de crecimiento de $A$. chilensis observados en este trabajo, con tasas de crecimiento elevadas sostenidas durante su breve período de vida, características propias de una especie colonizadora e intolerante a la sombra (Brienen \& Zuidema 2006). Los patrones de crecimiento observados en N. fernandeziana, sin embargo, se corresponden con los de una especie capaz de sobrevivir a elevadas intensidades de competencia y de experimentar bruscas liberaciones del crecimiento (Rentch et al. 2003). Las reducidas tasas de crecimiento inicial, correspondientes al período de desarrollo de los juveniles bajo un dosel arbóreo, y las bruscas liberaciones del crecimiento a lo largo de toda su vida, correspondientes a la apertura de claros en el dosel forestal adyacente, son características de las especies tolerantes a la sombra y típicas del bosque maduro (Rentch et al. 2003, Brienen \& Zuidema 2006). N. fernandeziana no puede establecerse bajo la sombra de árboles adultos de su propia especie (Vargas et al. 2010a), pero probablemente es capaz de establecerse bajo el dosel de otras especies menos frondosas, como es el caso de las especies invasoras.

Esta caracterización de las estrategias vitales propias de cada especie, realizada a partir de sus patrones de crecimiento, nos permite sugerir posibles prácticas de 
manejo para la conservación de la especie endémica $N$. fernandeziana, y el control o reducción de las poblaciones de la especie invasora A. chilensis en RC. En primer lugar, no parece recomendable la tala masiva sobre las poblaciones de $A$. chilensis, para evitar la generación de claros o espacios abiertos que favorezcan la recolonización de la especie. Estas prácticas facilitarían la regeneración vegetativa y/o por semilla de la especie exótica, incrementando su potencial de invasión (Quiroz et al. 2009). En segundo lugar, las poblaciones existentes de $A$. chilensis podrían favorecer el establecimiento natural o mediante plantación directa de $N$. fernandeziana en su sotobosque, ya que las condiciones de sombra creadas por $A$. chilensis facilitarían la supervivencia y el desarrollo juvenil de la especie endémica. Así, esta especie sería capaz de tolerar la competencia y sobrepasar el dosel de la especie invasora, con lo que llegaría un momento en que se formara un nuevo dosel de $N$. fernandeziana sobre el dosel previo, siendo gradualmente sustituida la especie invasora por la especie endémica.

\section{CONCLUSIONES}

La anatomía en corte transversal de la madera de las especies $N$. fernandeziana y A. chilensis estudiadas en RC permite la identificación y el análisis de sus anillos de crecimiento. Por lo tanto, es posible la realización de cualquier tipo de investigación basada en el estudio de anillos anuales de crecimiento, como la estimación de la edad de los árboles, estudios retrospectivos sobre la demografía y establecimiento de juveniles, y análisis a largo plazo de los patrones de crecimiento radial. N. fernandeziana es una especie tolerante a la sombra, con crecimiento inicial limitado, gran longevidad y gran capacidad de respuesta a la apertura de claros en el dosel de la Myrtiselva. Esta especie puede sobrevivir a una elevada intensidad de competencia y sus patrones de crecimiento radial pueden ser utilizados potencialmente para reconstruir a largo plazo la historia de perturbación del dosel forestal en RC. A. chilensis, sin embargo, es una especie colonizadora, intolerante a la sombra, con gran tasa de crecimiento durante toda su vida y escasa longevidad. En cuanto a su crecimiento radial y longevidad, la especie invasora se comporta en RC del mismo modo que en su área de distribución natural. Existe el potencial de utilizar las poblaciones actuales de A. chilensis en RC como una cobertura vegetal creadora de las condiciones que favorecerían el establecimiento y crecimiento juvenil de $N$. fernandeziana. Por lo tanto, con el objetivo de controlar la expansión de la especie invasora en la isla y de potenciar la recuperación de la especie endémica, se recomienda no actuar mediante talas masivas sobre los individuos de A. chilensis, sino favorecer el progresivo establecimiento de juveniles de $N$. fernandeziana a su sombra y efectuar un seguimiento a largo plazo de la evolución de las masas boscosas.
En cuanto a posibles estudios futuros, es preciso indicar que se requiere un diseño de muestreo más extenso en el espacio y sostenido en el tiempo de individuos de ambas especies, datando sus anillos de crecimiento con el propósito de cuantificar temporalmente su crecimiento anual y atribuir los patrones de crecimiento observados a algún factor ya sea ambiental (clima) o biológico (competencia, dinámica forestal). Esto permitiría conocer en detalle los efectos de la especie invasora sobre la endémica. También sería necesario realizar estudios dendroecológicos de las especies pioneras nativas de RC, y evaluar cómo las especies invasoras afectan su desarrollo. En cuanto a la especie invasora A. chilensis, se requiere mayor conocimiento sobre su capacidad de establecimiento y crecimiento diametral en su área de distribución natural en Chile continental.

\section{AGRADECIMIENTOS}

Agradecemos a Iván Leiva y Oscar Chamorro de CONAF en Juan Fernández por su valioso apoyo. A Juan Vera y Gianfranco Moris por la supervisión en terreno y el traslado de las muestras. Esta investigación fue financiada por el proyecto "High Resolution Hydroclimate Variability in the Central Andes of Chile and Argentina during the Last Millennium: a Tree-Ring and Modelling Perspective" (FONDECYT 1121106). La estadía de Vicente Rozas en la Universidad Austral de Chile, Valdivia, fue financiada por CONICYT mediante el Concurso Nacional "Atracción de Capital Humano Avanzado del Extranjero, Modalidad Estadías Cortas (MEC)" (Código 80130061).

\section{REFERENCIAS}

Allen, C.D., Savage, M., Falk, D.A., Suckling, K.F., Swetnam, T.W., Schulke, T., Stacey, P.B., Morgan, P., Hoffman, M., KLINGEL, J.T. 2002. Ecological restoration of southwestern ponderosa pine ecosystems: a broad perspective. Ecological Applications 12: 1418-1433.

Arellano-Cataldo, G. \& C. Smith-Ramírez. 2016. Establishment of invasive plant species in canopy gaps on Robinson Crusoe Island. Plant Ecology 217: 289-302.

Bernardello, G., Anderson, G.J., Stuessy, T.F., Crawford, D.J. 2011. The angiosperm flora of the Archipelago Juan Fernandez (Chile): Origin and dispersal. Canadian Journal of Botany 84(8): 1266-1281.

Brienen, R.J.W., Zuidema, P.A. 2006. Lifetime growth patterns and ages of Bolivian rain forest trees obtained by tree ring analysis. Journal of Ecology 94: 481-493.

Danton, P. 2004. Plantas silvestres de la isla Robinson Crusoe. Guía de reconocimiento. $1^{\text {a }}$ edición. CONAF. Viña del Mar, Chile. 194 pp.

Denardi, L., CARdoso. J.N. 2005. Anatomia ecológica da madeira de Blepharocalyx salicifolius (H. B. K.) Berg. Ciência Florestal 15(2): 119-127. 
Crecimiento de Nothomyrcia fernandeziana y Aristotelia chilensis en Robinson Crusoe: RoJas-BadiLla, M. ET AL.

Dias-Leme, C.L., Gasson, P., Lughadha, E.N. 1995. Wood anatomy of four Myrtaceae genera in the subtrive Myrciinae from South America. IAWA Journal 16(1): 87-95.

DíAZ-VAZ, J.E. 1979. Claves para la identificación de maderas de árboles nativos y cultivados en Chile. Bosque 3(1): 15-25.

Dirnböck T., Greimler, J., Lopez, P., Stuessy, T. 2003. Predicting Future Threats to the Native Vegetation of Robinson Crusoe Island, Juan Fernandez Archipelago, Chile. Conservation Biology 17(6): 1650-1659.

Fritts, H.C., Swetnam, T.W. 1989. Dendroecology: a tool for evaluating variations in past and present forest environments. Advances in Ecological Research 19: 111188.

Hechenleitner, P., Zamorano, C. 2005. Arboretum de la Universidad Austral de Chile: un modelo de conservación integral para nuestro país. Revista Chagual 3: 41-48.

Greimler, J., Lopez, P., Stuessy, T.F., Dirnbock, T. 2002. The vegetation of Robinson Crusoe Island (Isla Masatierra), Juan Fernandez Archipelago, Chile. Pacific Science 56(3): 263-284.

Hajek, E., Espinoza, G.A. 1987. Meteorología, climatología y bioclimatología de las Islas Oceánicas Chilenas. En: Castilla, J.C. (ed.), Islas Oceánicas Chilenas. pp. 55-83. Ediciones Universidad Católica de Chile, Santiago, Chile.

JoHow, F.R.A. 1896. Estudios sobre la flora de las islas de Juan Fernández. Imprenta Cervantes, Santiago de Chile Editorial. 287 pp.

Le Quesne, C., Rojas, M., Christie, D. 2014. Anillos de crecimiento de Austrocedrus chilensis: un archivo natural del cambio climático. Revista Chagual 12: 31-35.

Muñoz, A.A., GonzÁlez, M.E. 2006. Aristotelia chilensis. En: Donoso, C. (ed.), Las especies arbóreas de Chile y Argentina. Autoecología: 166-172. Editorial Marisa Cuneo, Valdivia, Chile.

Murillo-Aldana, J., Ruiz, E. 2011. Revalidación de Nothomyrcia (Myrtaceae), un género endémico del Archipiélago de Juan Fernández. Gayana Botanica 68(2): 129-134.

Murillo-A, J., Ruiz-P., E., Landrum, L.R., Stuessy, T.F., Barfuss, M.H.J. 2012. Phylogenetic relationships in Myrceugenia (Myrtaceae) based on plastid and nuclear DNA sequences. Molecular Phylogenetics and Evolution 62: 764-776.

Nowacki, G.J., ABRAms, M.D. 1997. Radial-growth averaging criteria for reconstructing disturbance histories from presettlement-origin oaks. Ecological Monographs 67: 225-249.

Quiroz, C., Pauchard, A., Marticorena, A., Cavieres, L. 2009. Manual de plantas invasoras del centro-sur de Chile. Universidad de Concepción, Concepción. 45 pp.

Rajni, N. Patel. 1989. Wood anatomy of the dicotyledons indigenous to New Zealand 18. Elaeocarpaceae, New Zealand Journal of Botany, 27: 3, 325-335.

Rentch, J.S., FaJvan, M.A., Hicks, R.R. 2003. Oak establishment and canopy accession strategies in five old-growth stands in the central hardwood forest region. Forest Ecology and Management 184: 285-297.

Rodrigues, S., Cardoso, J.N. 2010. Estudo anatômico do lenho de Myrcianthes cisplatensis (Camb.) O. Berg (Myrtaceae). Balduinia 24: 24-29.

Rojas-Badilla, M., Álvarez, C., Velásquez-Álvarez, G., Hadad, M., Le Quesne, C., Christie, D. (En prensa). Anomalías anatómicas en los anillos de crecimiento anuales de Austrocedrus chilensis en el límite norte de su distribución en Chile central. Gayana Botanica 74(2).

Rozas, V. 2003. Tree age estimates in Fagus sylvatica and Quercus robur: testing previous and improved methods. Plant Ecology 167: 193-212.

Rozas, V., Le Quesne, C., Muñoz, A., Puchi, P. 2016. Climate and growth of Podocarpus salignus in Valdivia, Chile. Dendrobiology 76: 3-11.

Smith-Ramírez, C., Arellano, G., Hagen, E., Vargas, R., Castillo, J., Miranda, A. 2013. El Rol de Turdus falcklandii (Aves: passeriforme) como dispersor de plantas invasoras en el archipiélago de Juan Fernández. Revista Chilena de Historia Natural 86: 33-48.

SpeER, J.H. 2010. Fundamentals of Tree Ring Research. University of Arizona Press, Tucson, AZ, USA. 333 pp.

Stuessy, T.F., Swenson, U., Crawford, D.J., Anderson, G.J., Silva, O.M. 1997. Plant Conservation in the Juan Fernandez Islands. Aliso 16: 89-102.

Swenson, U., Stuessy, T.F., Baeza, M., Crawford, D.J. 1997. New and historical plant introductions, and potential pests in the Juan Fernández, Chile. Pacific Science 51: 233-253.

Swetnam, T.W., Allen, C.D., Betancourt, J.L. 1999. Applied historical ecology: Using the past to manage for the future. Ecological Applications 9: 1189-1206.

Vargas, R., Cuevas, J.G., Le Quesne, C., Reif, A., Bannister, J. 2010a. Spatial distribution and regeneration strategies of the main forest species on Robinson Crusoe Island. Revista Chilena de Historia Natural 83: 349-363.

VARGas, R., FAúndez, M.J. 2010. Los bosques de Robinson Crusoe: endemismo para conservar. Gestión Ambiental 20: 19-26. (http://revista.bosquenativo.cl/volumenes/45/3_vargas. htm).

Vargas, R., Gärtner, S., Alvarez, M., Hagen, E., Reif, A. 2013. Does restoration help the conservation of the threatened forest of Robinson Crusoe Island? The impact of forest gap attributes on endemic plant species richness and exotic invasions. Biodiversity Conservation 22: 1283-1300.

Vargas, R., Reif, A., Danton, P. 2010b. Los bosques de la isla Robinson Crusoe: el tesoro en peligro. Revista Bosque Nativo 45: 3-8.

Vargas, R., Smith-Ramírez, C., González, C., Fernández, M. 2014. Reserva de la Biosfera Archipiélago Juan Fernández: endemismo para conservar. En: Moreira-Muñoz, A., Borsdorf, A. (eds.), Reservas de la Biosfera de Chile: Laboratorios para la Sustentabilidad. Vol. 17, pp. 126-143. Academia de Ciencias Austriaca, Pontificia Universidad Católica de Chile, Instituto de Geografía, Santiago, Chile.

World Conservation Monitoring Centre. 1998. Myrceugenia fernandeziana. The IUCN Red List of Threatened Species 1998: e.T34177A9847804. http://dx.doi.org/10.2305/ IUCN.UK.1998.RLTS.T34177A9847804.en. Downloaded on 16 August 2016. 